\title{
A case of fatal idiosyncratic reaction to the designer drug 3,4-methylenedioxypyrovalerone (MDPV) and review of the literature
}

\author{
Brigitte Desharnais $^{\mathrm{a}, \mathrm{b}, *}$, Yann Dazéc ${ }^{\mathrm{c}}$, Laura M. Huppertz ${ }^{\mathrm{d}}$, Pascal Mireault ${ }^{\mathrm{a}}$, \\ Cameron D. Skinner ${ }^{\mathrm{b}}$ \\ ${ }^{a}$ Department of Toxicology, Laboratoire de sciences judiciaires et de médecine légale \\ 1701 Parthenais Street, Montréal, Québec, Canada H2K 3S7 \\ ${ }^{b}$ Department of Chemistry \& Biochemistry, Concordia University \\ 7141 Sherbrooke Street West, Montréal, Québec, Canada H4B 1R6 \\ ${ }^{c}$ Department of Forensic Pathology, Laboratoire de sciences judiciaires et de médecine légale \\ 1701 Parthenais Street, Montréal, Québec, Canada H2K 3S7 \\ ${ }^{d}$ Institute of Forensic Medicine, Department of Forensic Toxicology, Medical Center, \\ Faculty of Medicine, University of Freiburg, Albertstrasse 9, 79104, Freiburg, Germany
}

\begin{abstract}
The stimulant designer drug 3,4-methylenedioxypyrovalerone (MDPV) was first synthesized by Boehringer Ingelheim in 1969 and introduced on the black market in 2006. Only a small number of fatal intoxication cases have been reported in the literature, all with significant blood MDPV concentrations. In this report, we describe one fatality attributed to an idiosyncratic reaction to MDPV. The victim displayed agitation, violent behavior and delirium followed by cardiac arrest. Hyperthermia was observed at the hospital. The MDPV cardiac and femoral blood concentrations were $6 \mathrm{ng} / \mathrm{mL}$. The presence of excited delirium syndrome and MDPV, a drug with a pharmacology similar to cocaine, leads to the conclusion that the victim suffered a fatal adverse reaction to MDPV. This is the first published case of idiosyncratic reaction to MDPV.
\end{abstract}

\section{Introduction}

In order to circumvent existing legislation on controlled substances, derivatives of known psychoactive substances began to appear on the black market as "designer drugs" in the mid-2000s [1, 2]. One popular family of designer drugs are the synthetic cathinones or "bath salts". 3,4-methylenedioxypyrovalerone

*Author to whom correspondence should be addressed.

Email: brigitte.desharnais@msp.gouv.qc.ca

Post-print of the article published in Forensic Science, Medicine and Pathology

July 1, 2017 
(MDPV) is a member of this family: a $\beta$-keto phenylalkylamine, derivative of the natural alkaloid cathinone found in the plant Catha edulis (khat) [1, 3]. MDPV, initially synthesized by Boehringer Ingelheim in 1969 [1], was first detected in Japan in 2006, and subsequently was increasingly found throughout the world $[4,5]$. In 2011, the Drug Enforcement Agency of the United States (DEA) scheduled MDPV on a temporary emergency basis [6, 7]. Since this time, the prevalence of MDPV in synthetic cathinones decreased in favor of unregulated compounds.

Synthetic cathinones are stimulants consumed for the effects of increased alertness and energy, euphoria, excitement, improved mood, sociability and intensification of sensory experience (mild empathogenic effects) [5]. Undesired cardiac and neurological effects include tachycardia, hypertension, cardiac arrest, agitation, aggressive and combative behavior, disorientation, confusion and paranoia $[1,5,6,7,8,9,10]$.

The physical form of MDPV is a white, tan, gray, yellow or brown odorless powder $[1,6]$ and is often mixed with caffeine and lidocaine or benzocaine [5]. It is marketed as "bath salt" (in the US) or "plant feeder" (in Europe) and sold under 50 different known brand names on the Internet or in head shops, convenience stores, gas stations and tobacco shops $[5,6,11,12]$. Its main modes of ingestion are oral, intravenous, insufflation (snorting) and inhalation (smoking) $[1,5,6,8,13,14,15]$. Doses of 5 to $30 \mathrm{mg}$ in a single ingestion are typical $[1,5,8]$, with effects lasting 1 to $3 \mathrm{~h}[10]$.

Although several clinical cases of consumption of, or intoxication by, MDPV have been reported $[4,9,12,13,15,16,17,18,19,20,21]$, there are only a few fatal MDPV intoxication cases published with toxicology results $[3,6,10,14,22,23]$. In all of these cases, the MDPV concentration was elevated $(>80 \mathrm{ng} / \mathrm{mL}$ in whole blood). We present here a case of fatal idiosyncratic reaction to MDPV, a radically different type of MDPV intoxication than what has been previously published.

\section{Case history}

\subsection{Background}

The victim was a 42 year old Native American male that had been incarcerated for several months prior to the event. In the early afternoon, the man was standing in front of his cell, talking incoherently. He then approached the guards' post and became hysterical and disorganized. Prison personnel attempted to 
physically control him, but he fought them vigorously. He was eventually cuffed at the wrists and ankles and put into a padded cell, lying on his side. 27 minutes later, the man was in cardiorespiratory arrest and resuscitation maneuvers were started. Upon arrival at the hospital, 1 h19 into the event, the victim's rectal temperature was $39.3^{\circ} \mathrm{C}$. His death was pronounced $1 \mathrm{~h} 40$ after the beginning of the event. Since the victim was in cardiac arrest upon arrival at the hospital, no biochemistry or toxicology screen was performed.

\subsection{Autopsy findings}

The deceased was $182 \mathrm{~cm}$ tall and weighed $156 \mathrm{~kg}$. The external exam revealed the presence of linear abrasions and light bruises at the wrists and ankles, most likely due to the cuffs placed on the victim. Also, numerous bruises were observed on the four limbs, which could have resulted from confinement of the victim by the prison personnel or have been auto-inflicted. A linear abrasion on the shoulder blade and abrasions on the knees were also observed. No other significant traumatic lesions were apparent on external examination.

The internal exam revealed the presence of left pleural adhesions. The heart weighed $520 \mathrm{~g}$ and revealed a slight left ventricle hypertrophy, without any congenital anomaly, valvular or coronary disease. The lungs (right $820 \mathrm{~g}$, left $870 \mathrm{~g}$ ) were heavy and congested, which was confirmed through histological analysis. The liver weighed $1780 \mathrm{~g}$ and was affected by steatosis without associated cirrhosis, which was also confirmed by the histological exam. The urinary bladder contained $80 \mathrm{~mL}$ of urine. The full length of the gastro-intestinal tract was opened and no intra-luminal body was found. Gastric contents $(60 \mathrm{~mL})$ were a brownish liquid with beige, black and green particles. Rib fractures attributed to CPR were observed. No cranial, spine, hip or limb fractures were seen. No other anatomic lesions were observed.

\subsection{Toxicological analysis}

Peripheral blood from the femoral vein, cardiac blood (pooled from both ventricles), urine, gastric contents and vitreous humor were recovered for toxicological analyses. Toxicology included cyanide screening, ethanol and volatile compound determination, immunological screening (cocaine, opiates, PCP, amphetamines, cannabinoids, barbiturates, salicylates, benzodiazepines, acetaminophen, paracetamol and methadone) as well as gas-chromatography-mass spectrometry (GC-MS-NPD) screening and liquid chromatography-tandem mass spectrometry (LC-MS/MS) quantification. All of these methods (unpublished) are validated, and the quantitative methods are accredited under ISO 17025/CAN-P 
1578 standards. To carry out the LC-MS/MS analysis, all matrices were extracted with a protein precipitation protocol using a mixture of acetonitrile and acetone as the precipitating agent. Results of the toxicological investigation are shown in Table 1.

Table 1: Postmortem toxicological analysis results

\begin{tabular}{|l|l|l|l|l|l|l|}
\hline \multirow{2}{*}{ Biological Matrix } & \multicolumn{5}{|c|}{ Concentration (ng/mL) } \\
\cline { 2 - 8 } & MDV & Mirtazapine & THC & THC-OH & THC-COOH & 7-amino-clonazepam \\
\hline Femoral Blood & 6 & 17 & 4 & 4 & 27 & 2 \\
\hline Cardiac Blood & 6 & 26 & 1 & 2 & 38 & 3 \\
\hline Urine & 166 & 290 & ND & 1 & 290 & 5 \\
\hline Gastric Contents & 19 & 180 & 273 & 61 & 64 & ND \\
\hline Vitreous Humour & 5 & 2 & ND & ND & 1 & ND \\
\hline
\end{tabular}

ND: not detected

Also detected in cardiac blood, femoral blood and urine were the metabolites demethylenyl-methyl-MDPV, N-desmethylmirtazapine and THC-COOH glucuronide. According to medical records, the victim had a prescription for mirtazapine and clonazepam, explaining the presence of these two compounds in the toxicology results.

By taking into account circumstances surrounding death (agitation, violent behavior, hyperthermia) and toxicology results, the cause of death was ruled to be complications of an adverse reaction to consumption of MDPV by the victim. It was also noted that the pre-existing medical condition of the victim (obesity, slight cardiac hypertrophy) may have contributed to death.

Subsequent toxicological screening by liquid chromatography-time of flight mass spectrometry (LC-QToF) initially suggested the presence of 4-acetoxy-DMT (4-AcO-DMT) in cardiac blood and urine via a library hit. 4-acetoxy-DMT (also known as psilacetin and O-acetylpsilocin) is a synthetic pro-drug of the hallucinogenic compound psilocin [24]. However, further analysis using reference material confirmed this as a false positive, since retention time did not match and specific fragments were absent. 


\section{Discussion}

A certain number of MDPV intoxication cases have already been published in the literature (see Table 2). In all published reports, the MDPV concentration in blood is more elevated than what was observed in the case presented here. Indeed, all but one case show MDPV concentrations at least 10 times higher than the $6 \mathrm{ng} / \mathrm{mL}$ found in the cardiac and femoral blood of the victim. Although an MDPV overdose (in the strictest sense of the term) seems unlikely here, an adverse reaction to MDPV is a probable mechanism of death.

MDPV is a dopamine and norepinephrine reuptake inhibitor acting as a stimulant $[1,7,9,22]$. In vitro transporter assays performed on brain synaptosomes show that MDPV functions as a monoamine uptake pure blocker (like cocaine) and not as a monoamine transporter substrate (like amphetamine) [25]. The pharmacology of MDPV is thus closer to cocaine than MDMA, as was previously thought.

It is well known that there is no "safe" concentration for cocaine, since virtually any concentration can trigger an adverse reaction and ultimately death [26]. Given the similarity between the pharmacology of MDPV and cocaine, it would seem logical that a similar potential for adverse reactions exists with MDPV.

Stimulant drug use, particularly cocaine, is a known trigger of excited delirium syndrome (ExDS), even at recreational dosages [27]. Excited delirium syndrome is characterized by an excited or agitated state, delirium, sometimes complemented by tachycardia and hyperthermia followed by cardiac arrest [3, 27]. The behavior reported by prison personnel as well as the hyperthermia highlighted at the hospital both fit the ExDS profile. The exact mechanism of this potentially lethal side effect is unknown. The observed hyperthermia is thought to result from dysregulation of the dopaminergic pathways in the hypothalamus [3] and has been reported in an elevated number of MDPV intoxications and adverse reactions $[3,4,6,9,16,17,23]$. The presence of ExDS adds to the profile of an adverse reaction to a stimulant. It is worth noting that the presence of mirtazapine, even at therapeutic concentrations, might have contributed to the elevation of serotonin levels and thus the reported agitation of the victim. 
Table 2: Previously published MDPV fatal intoxications

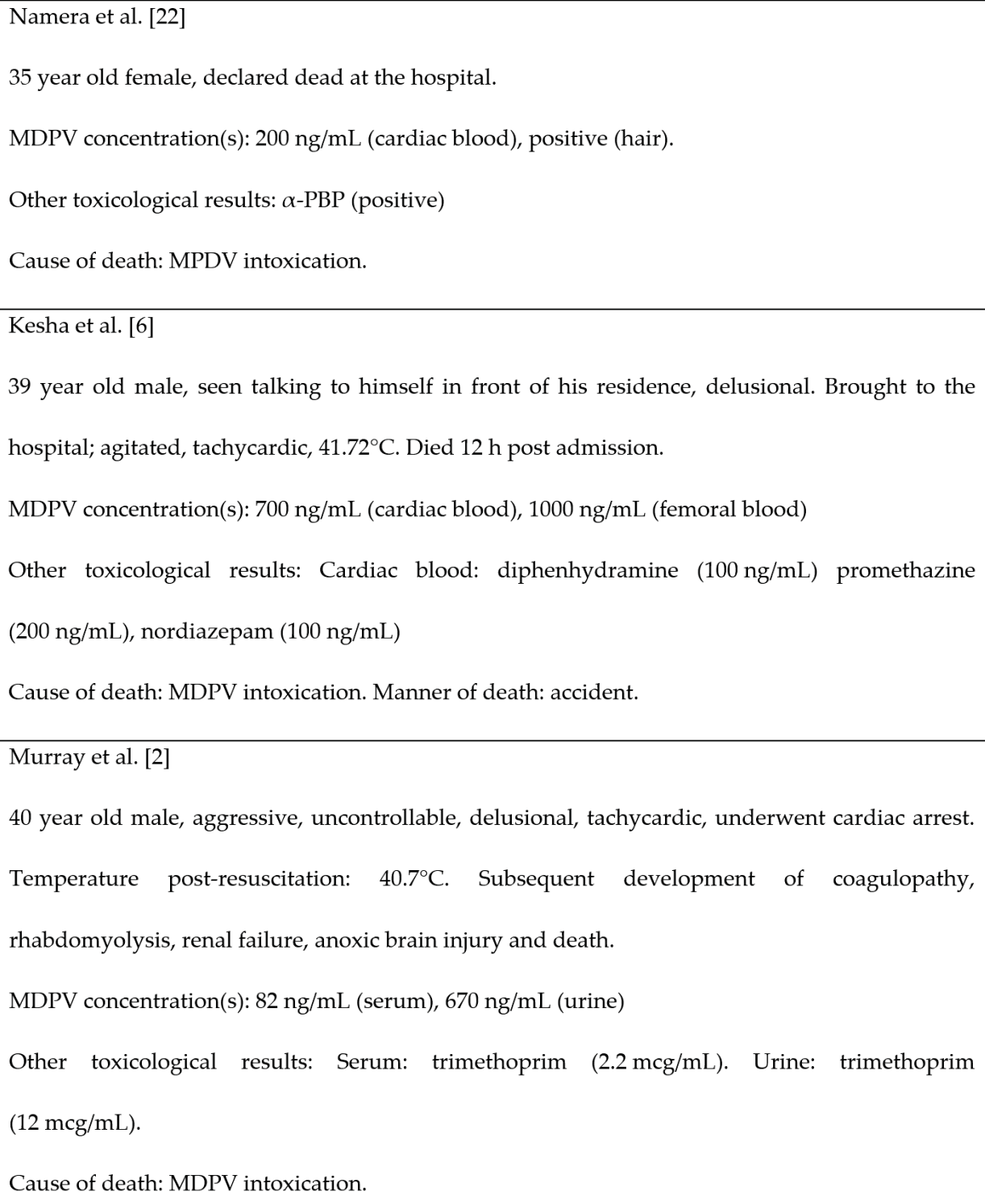




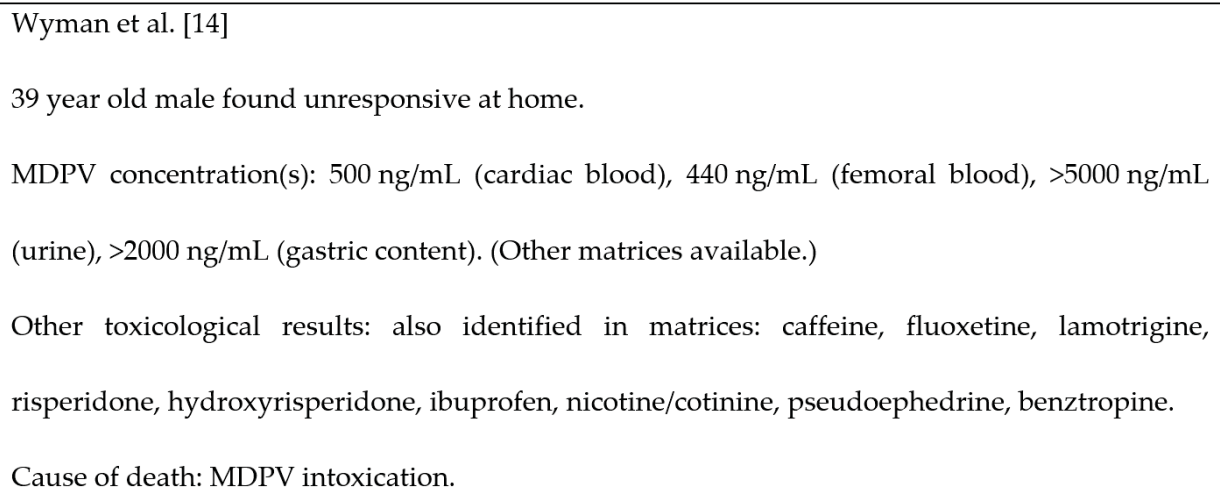


Demethylenyl-methyl-MDPV (also called methyl cathecol pyrovalerone [2]) has been previously identified in studies of in vitro human liver microsomes and in vivo rat MDPV metabolism [2,28]. It is hypothesized to result from the methylation of the initial demethylenyl-MDPV metabolite produced by CYP 1A2, CYP 2C19 and CYP 2D6 [28] enzymes. The lack of additional metabolite standards limited LC-MS/MS analysis for identification and quantification of other metabolites besides demethylenyl-methyl-MDPV in the matrices.

In the case reported here, several elements point towards a case of fatal idiosyncratic reaction to the stimulant designer drug MDPV, such as excited delirium syndrome with hyperthermia, low concentrations of MDPV in blood and the similarity of the pharmacology of MDPV with cocaine. This is a category of MDPV intoxication previously unreported in the literature.

\section{Key points}

1. 3,4-methylenedioxypyrovalerone (MDPV) is a stimulant designer drug with a pharmacology similar to cocaine.

2. Excited delirium syndrome (ExDS) is characterized by agitation, delirium, tachycardia and hyperthermia. A common trigger is stimulant drug consumption.

3. Presence of ExDS with recreational MDPV concentrations should be considered as indications of a potential adverse reaction to MDPV.

\section{Acknowledgements}

The authors would like to thank Anne-Marie Faucher for the initial toxicological analysis, Cynthia Côté and Julie Laquerre for subsequent xenobiotic quantification by LC-MS/MS, as well as Dr. Pierre Brochu, coroner, for access to the medical file and authorization to publish this case report.

\section{Compliance with ethical standards}

\subsection{Funding}

This study was funded by the Laboratoire de sciences judiciaires et de médecine légale. Brigitte Desharnais and Cameron D. Skinner gratefully acknowledge support of the National Sciences and Engineering Research Council of Canada. Brigitte Desharnais also gratefully acknowledges the support of the Fonds de recherche du Québec - Nature et technologies. 


\subsection{Conflict of interest}

The authors declare that they have no conflict of interest.

\subsection{Ethical approval}

All procedures performed in studies involving human participants were in accordance with the ethical standards of the institutional and/or national research committee and with the 1964 Helsinki declaration and its later amendments or comparable ethical standards. Consent to publish this case report was obtained from the coroner.

\section{References}

[1] M. Coppola, R. Mondola, 3, 4-methylenedioxypyrovalerone (MDPV): chemistry, pharmacology and toxicology of a new designer drug of abuse marketed online, Toxicology Letters 208 (2012) 12-15.

[2] S. Strano-Rossi, A. B. Cadwallader, X. de la Torre, F. Botrè, Toxicological determination and in vitro metabolism of the designer drug methylenedioxypyrovalerone (MPDV) by gas chromatography/mass spectrometry and liquid chromatography/quadrupole time-of-flight mass spectrometry, Rapid Communications in Mass Spectrometry 24 (2010) 2706-2714.

[3] B. L. Murray, C. M. Murphy, M. C. Beuhler, Death following recreational use of designer drug "bath salts" containing 3, 4-methylenedioxypyrovalerone (MDPV), Journal of Medical Toxicology 8 (2012) 69-75.

[4] H. A. Borek, C. P. Holstege, Hyperthermia and multiorgan failure after abuse of "bath salts" containing 3, 4-methylenedioxypyrovalerone, Annals of Emergency Medicine 60 (2012) 103-105.

[5] J. B. Zawilska, J. Wojcieszak, Designer cathinones - an emerging class of novel recreational drugs, Forensic Science International 231 (2013) 42-53.

[6] K. Kesha, C. L. Boggs, M. G. Ripple, C. H. Allan, B. Levine, R. Jufer-Phipps, S. Doyon, P. Chi, D. R. Fowler, Methylenedioxypyrovalerone ("bath salts"), related death: case report and review of the literature, Journal of Forensic Sciences 58 (2013) 1654-1659.

[7] J. Jerry, G. Collins, D. Streem, Synthetic legal intoxicating drugs: the emerging "incense" and "bath salt" phenomenon, Cleveland Clinic Journal of Medicine 79 (2012) 258-264.

[8] E. A. Ross, M. Watson, B. Goldberger, "Bath salts" intoxication, New England Journal of Medicine 365 (2011) 967-968.

[9] H. Regunath, V. K. Ariyamuthu, P. Dalal, M. Misra, Bath salt intoxication causing acute kidney injury requiring hemodialysis, Hemodialysis International 16 (2012) S47-S49.

[10] L. J. Marinetti, H. M. Antonides, Analysis of synthetic cathinones commonly found in bath salts in human performance and postmortem toxicology: method development, drug distribution and interpretation of results, Journal of Analytical Toxicology 37 (2013) 135146.

[11] M. J. Swortwood, D. M. Boland, A. P. DeCaprio, Determination of 32 cathinone derivatives and other designer drugs in serum by comprehensive LC-QQQ-MS/MS analysis, Analytical and Bioanalytical Chemistry 405 (2013) 1383-1397.

[12] H. A. Spiller, M. L. Ryan, R. G. Weston, J. Jansen, Clinical experience with and analytical confirmation of "bath salts" and "legal highs" (synthetic cathinones) in the United States, Clinical Toxicology 49 (2011) 499-505. 
[13] S. Férec, M. Bretaudeau-Deguigne, B. Lelièvre, D. Boels, C. Bruneau, I. Leborgne, P. Harry, B. Diquet, A. Turcant, Expositions récréatives de 8 patients aux nouvelles drogues de synthèse obtenues sur Internet: à propos de 3, 4-méthylènedioxypyrovalérone (MDPV) et de méthoxétamine (MXE), Annales de Toxicologie Analytique 25 (2013) 47-56.

[14] J. F. Wyman, E. S. Lavins, D. Engelhart, E. J. Armstrong, K. D. Snell, P. D. Boggs, S. M. Taylor, R. N. Norris, F. P. Miller, Postmortem tissue distribution of MDPV following lethal intoxication by "bath salts", Journal of Analytical Toxicology 37 (2013) 182-185.

[15] P. Kriikku, L. Wilhelm, O. Schwarz, J. Rintatalo, New designer drug of abuse: 3, 4-Methylenedioxypyrovalerone (MDPV). Findings from apprehended drivers in Finland, Forensic Science International 210 (2011) 195-200.

[16] J. Mugele, K. A. Nañagas, L. M. Tormoehlen, Serotonin syndrome associated with MDPV use: a case report, Annals of Emergency Medicine 60 (2012) 100-102.

[17] S. Fröhlich, E. Lambe, J. O'Dea, Acute liver failure following recreational use of psychotropic "head shop" compounds, Irish Journal of Medical Science 180 (2011) 263-264.

[18] S. L. Thornton, R. R. Gerona, C. A. Tomaszewski, Psychosis from a bath salt product containing flephedrone and MDPV with serum, urine, and product quantification, Journal of Medical Toxicology 8 (2012) 310-313.

[19] T. M. Penders, R. Gestring, Hallucinatory delirium following use of MDPV: "Bath Salts", General Hospital Psychiatry 5 (2011) 525-526.

[20] E. Bertol, F. Mari, R. B. Berto, G. Mannaioni, F. Vaiano, D. Favretto, A mixed MDPV and benzodiazepine intoxication in a chronic drug abuser: determination of MDPV metabolites by LC-HRMS and discussion of the case, Forensic Science International 243 (2014) 149 155.

[21] H. C. Scarface, R. Dove, W. Dove, Emergency Department Visits After Use of a Drug Sold as "Bath Salts"-Michigan, November 13, 2010-March 31, 2011, Morbidity and Mortality Weekly Report (2011).

[22] A. Namera, S. Urabe, T. Saito, A. Torikoshi-Hatano, H. Shiraishi, Y. Arima, M. Nagao, A fatal case of 3, 4-methylenedioxypyrovalerone poisoning: coexistence of $\alpha$ pyrrolidinobutiophenone and $\alpha$-pyrrolidinovalerophenone in blood and/or hair, Forensic Toxicology 31 (2013) 338-343.

[23] A. C. Young, E. S. Schwarz, L. I. Velez, M. Gardner, Two cases of disseminated intravascular coagulation due to "bath salts" resulting in fatalities, with laboratory confirmation, The American Journal of Emergency Medicine 31 (2013) 445-e3.

[24] D. E. Nichols, S. Frescas, Improvements to the synthesis of psilocybin and a facile method for preparing the O-acetyl prodrug of psilocin, Synthesis (1999) 935-938.

[25] M. H. Baumann, J. S. Partilla, K. R. Lehner, E. B. Thorndike, A. F. Hoffman, M. Holy, R. B. Rothman, S. R. Goldberg, C. R. Lupica, H. H. Sitte, et al., Powerful cocaine-like actions of 3, 4-methylenedioxypyrovalerone (MDPV), a principal constituent of psychoactive "bath salts" products, Neuropsychopharmacology 38 (2013) 552.

[26] J. R. Gill, Practical toxicology for the forensic pathologist, in: T. M. (Ed.), Forensic Pathology Reviews, volume 2, Humana Press, Totowa, New Jersey, United States, 2005, pp. 243-269.

[27] G. M. Vilke, M. L. DeBard, T. C. Chan, J. D. Ho, D. M. Dawes, C. Hall, M. D. Curtis, M. W. Costello, D. C. Mash, S. R. Coffman, et al., Excited delirium syndrome (ExDS): defining based on a review of the literature, The Journal of Emergency Medicine 43 (2012) 897-905.

[28] M. R. Meyer, P. Du, F. Schuster, H. H. Maurer, Studies on the metabolism of the $\alpha$ pyrrolidinophenone designer drug methylenedioxy-pyrovalerone (MDPV) in rat and human urine and human liver microsomes using GC-MS and LC-high-resolution MS and its detectability in urine by GC-MS, Journal of Mass Spectrometry 45 (2010) 1426-1442. 